\title{
The British Society for Cardiovascular Research Autumn 2019 Meeting Incorporating the Bernard and Joan Marshall Research Awards
}

\author{
Sanjay Sinha ${ }^{1,2} \cdot$ David J. Grieve ${ }^{2,3}$ \\ Published online: 15 February 2020 \\ (C) Springer Science+Business Media, LLC, part of Springer Nature 2020
}

On behalf of the British Society for Cardiovascular Research (BSCR; https://bscr.org), we are delighted to introduce the second special issue of Cardiovascular Drugs and Therapy focussed on proceedings of our Autumn 2019 Meeting, hosted by Emmanuel College, University of Cambridge, which includes presented abstract communications [1] and four short review articles from the Marshall Early Career Investigator Award finalists [2-5]. These awards are supported by a generous bequest to the BSCR by Bernard and Joan Marshall which enables the society to honour the best British and international cardiovascular research, through the Early Career Investigator Awards as well as the Distinguished Investigator Lecture, which is presented annually at the BSCR Autumn Meeting by a world-leading cardiovascular scientist. This year, which marked the 10th anniversary of these prestigious awards, we were delighted to be joined by Peter Marshall, Bernard's brother, and his wife Rita, for both the prize session and the society dinner at which they presented the awards. The BSCR is extremely grateful to the Marshall family for their significant support over the past 10 years which has enabled the society to particularly recognise and encourage the next generation of cardiovascular scientists.

David J. Grieve

d.grieve@qub.ac.uk

1 Wellcome Trust-Medical Research Council Cambridge Stem Cell Institute, University of Cambridge, Cambridge, UK

2 British Society for Cardiovascular Research, London, UK

3 Queen's University Belfast, Wellcome-Wolfson Institute for Experimental Medicine, Belfast BT9 7AE, UK
In this regard, it seems timely to highlight the significant achievements of our previous Bernard and Joan Marshall Young Investigator Award (as it was known until 2017) winners. Dr. Alexander Lyon (2010) is a Senior Lecturer in Cardiology, Imperial College London, and Consultant Cardiologist at the Royal Brompton Hospital, where he is the theme leader for NIHR-funded heart failure research. Dr. Joseph Burgoyne (2011) is a Senior Lecturer at King's College London. Dr. Christine Cheung (2012) is an Assistant Professor at Lee Kong Chian School of Medicine, Nanyang Technological University, Singapore, and member of the Executive Committee of the Stem Cell Society of Singapore. Dr. Margaux Horn (2013) is a Lecturer in Bioscience at Keele University, specialising in cardiovascular physiology and pharmacology. Dr. Edward Chouchani (2014) is an Assistant Professor at Harvard Medical School. Dr. Dharini Iyer (2015) leads the cell therapy development team at Senti Biosciences in San Francisco. Dr. Victoria Mascetti (2016) is a Postdoctoral Fellow in stem cell biology and regenerative medicine at Stanford University. Dr. Johannes Bargehr (2017) is a Cardiology Academic Clinical Fellow at the University of Cambridge, with a specialty interest in regenerative cardiovascular medicine. Dr. Marwa Mahmoud (2018) is a Postdoctoral Fellow at City University, New York, researching mechanotransduction and endothelial dysfunction and was recently awarded the Ruth L. Kirschtein Fellowship from the National Institute of Health. Together, these achievements highlight the calibre of our previous Bernard and Joan Marshall Young Investigators and the importance of supporting such talent through this competition, and we look forward to reporting on the career successes of future winners. 


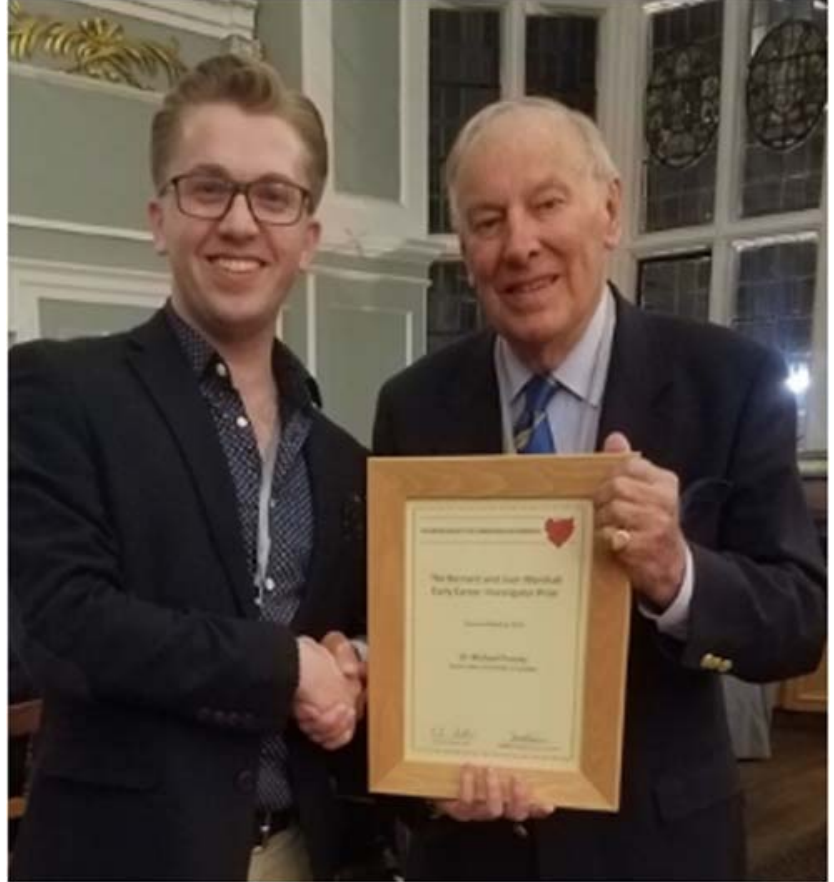

Mr Michael Preedy being presented with the Bernard and Joan Marshall Early Career Investigator Award by Peter Marshall

The Bernard and Joan Marshall Early Career Investigator Award was contested by four shortlisted finalists who have each contributed a short review article to this special issue of Cardiovascular Drugs and Therapy [2-5] based on original research published in leading journals including Circulation Research, Genetics in Medicine, NMR in Biomedicine, and Proceedings of the National Academy of Sciences of the United States of America. Mr. Michael Preedy, a final year British Heart Foundation-funded $\mathrm{PhD}$ student from the research group of Professor Adrian Hobbs at The William Harvey Research Institute, Queen Mary University of London, received the Bernard and Joan Marshall Early Career Investigator Award for his exciting work on the patho-physiological roles of guanylyl cyclases (GCs) within the heart. Impaired nitric oxide (NO)/guanylyl cyclase (GC)/ cyclic guanosine-3',5'-monophosphate (cGMP) signalling, along with upregulation of cyclic nucleotide-hydrolysing phosphodiesterase (PDE) isozymes, contributes to the pathogenesis of heart failure. However, the role of PDE2 has so far been relatively poorly investigated, and in his studies, $\mathrm{Mr}$. Preedy showed that selective inhibition of PDE2 in mice using either a chemical or genetic approach preferentially enhances $\mathrm{NO} / \mathrm{GC} / \mathrm{cGMP}$ signalling, leading to preservation of cardiac structure and function in a pressure-overload heart failure model. These findings substantiate the suggestion that PDE2-targeting drugs could be a new and productive approach to target both left ventricular hypertrophy and failure. The three runners-up in the Bernard and Joan Marshall Early Career Investigator Award final provided keen competition for Mr. Preedy and showcased a diverse range of cutting edge research. Dr. Andrew Lewis, a physician who recently completed his $\mathrm{PhD}$ at the University of Oxford, spoke about his work on hyperpolarised magnetic resonance. This emerging imaging technology uses very short-lived hyperpolarised contrast agents to provide increases in signal-to-noise ratio of 4 or 5 orders of magnitude. Dr. Lewis presented data on [113C]pyruvate imaging to show that in animal models, myocardial infarction causes an unexpected and intense [113C]lactate signature from the injured myocardial segments which has an immunological origin. Blockade of glycolysis with 2-deoxyglucose in activated cells and after rodent myocardial infarction normalised the hyperpolarised [113C]lactate signal and also caused dose-dependent improvement in IL-1 $\beta$ expression in infarcted tissue. Cine magnetic resonance imaging demonstrated improvements in systolic function in 2-deoxyglucose-treated rats at 3 months. Dr. Kerstin Timm, a British Heart Foundation Intermediate Basic Science Research Fellow and Lecturer in Metabolism at the University of Oxford, presented her work on 'Early detection of doxorubicin-induced heart failure via its in vivo
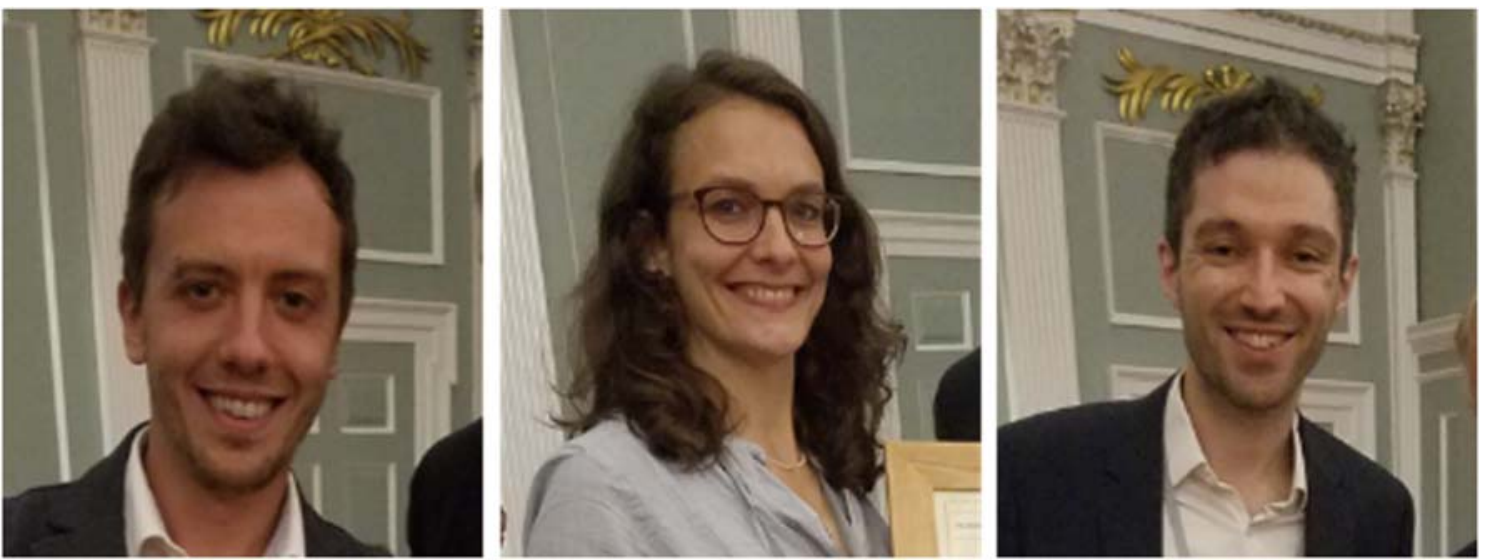

Dr Francesco Mazzarotto (on the left), Dr Kerstin Timm, and Dr Andrew Lewis (on the right), who were runners-up in the Bernard and Joan Marshall Early Career Investigator Award 


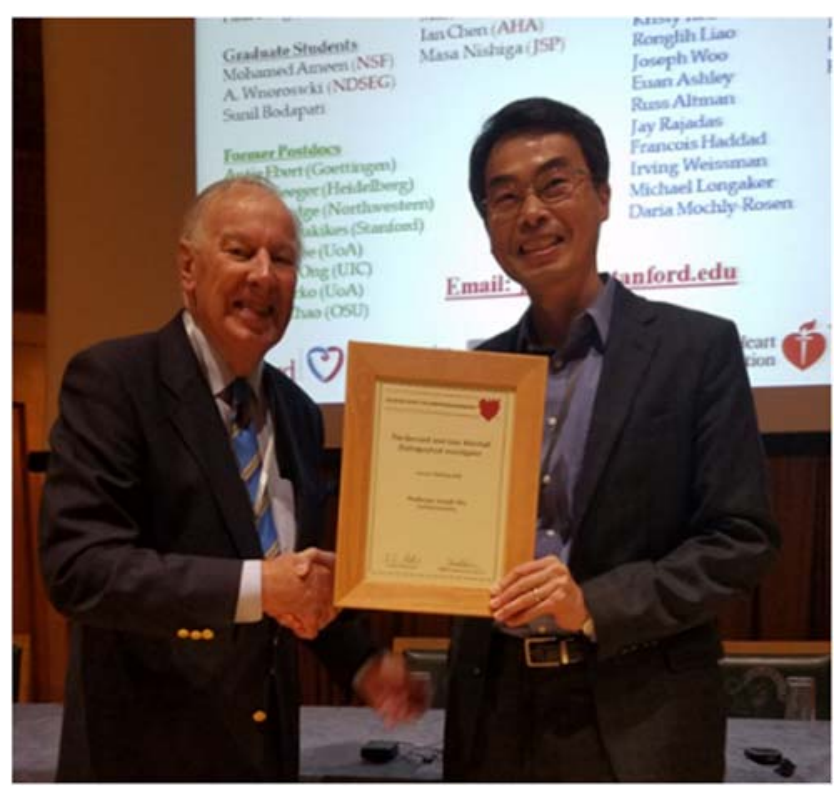

Professor Joseph Wu being presented with the Bernard and Joan Marshall Distinguished Lecturer Award by Peter Marshall

metabolic signature'. Doxorubicin is an anthracycline antitumour agent with dose-related cardiotoxicity in an unpredictable subset of patients. Dr. Timm's studies used hyperpolarised magnetic resonance imaging to detect early metabolic changes that characterise this condition and precede cardiac functional decline in a rat model. She also showed that 5-aminoimidazole-4-carboxamide 1- $\beta$-D-ribofuranoside (AICAR), an activator of AMPK that promotes mitochondrial biogenesis and fatty acid oxidation, rescued cardiac functional decline in experimental doxorubicin-induced heart failure. Dr. Francesco Mazzarotto, who is currently a Postdoctoral Fellow at the University of Florence, presented his recent work on genetic diagnosis using a novel metric that he called 'diagnostic effectiveness' - a score estimating the relative effectiveness of a given gene in a diagnostic panel for hypertrophic cardiomyopathy. Dr. Mazzarotto showed that next-generation sequencing allowed additional sensitivity in screening, but that this was achieved predominantly by focussing on genes incontrovertibly associated with hypertrophic cardiomyopathy or its mimics, with limited or no benefit from wider array of genes. We hope that you enjoy reading the short review articles contributed by all four of the Bernard and Joan Marshall Early Career Investigator Award finalists, relevant to their areas of research, published in this special issue of Cardiovascular Drugs and Therapy.

We were delighted to honour Professor Joseph Wu from Stanford University with the Bernard and Joan Marshall Distinguished Investigator Award. This award is presented annually to a researcher that in the opinion of the BSCR committee has made distinguished contributions to cardiovascular research over many years and exemplifies the highest academic and professional standards in our field. The Bernard and Joan Marshall Distinguished Investigator receives an honorarium and is invited to present a keynote lecture at the BSCR Autumn Meeting. Professor Wu graduated from Yale School of Medicine where he originally developed his love of research by combining cardiovascular pharmacology and imaging in large animal models. He subsequently trained in internal medicine and cardiology at the University of California Los Angeles, before undertaking a $\mathrm{PhD}$ in the Department of Molecular Pharmacology with Dr. Sanjiv Gambhir. Here he developed molecular imaging technology to track gene and cardiac stem cell therapy in vivo using bioluminescence or positron-emission tomography, studies that he still rates as among his favourites and a defining period in his development as a scientist. Professor $\mathrm{Wu}$ was appointed as an Instructor in Medicine at Stanford University in 2004 and rapidly progressed to his current position as Director of the Stanford Cardiovascular Institute and the Simon H. Stertzer, MD, Professor of Medicine and Radiology at the Stanford School of Medicine. His laboratory focusses on unravelling biological mechanisms of patient-specific and disease-specific induced pluripotent stem cells (iPSCs). Professor Wu believes that iPSC-derived cells can be used to integrate the thousands of genetic variants that influence tissue behaviour and disease development in individual patients, thus representing a powerful tool for clinical prediction and patient-specific studies. His main goals are to (i) understand basic cardiovascular disease mechanisms, (ii) accelerate drug discovery and screening, (iii) develop the 'clinical trial in a dish' concept, and (iv) implement precision cardiovascular medicine for disease prevention and treatment of patients. Professor $\mathrm{Wu}$ has published $>350$ manuscripts with 37,000 citations and an H-index of 95 according to Google Scholar. His research achievements have led to numerous awards including the Presidential Early Career Award for Scientists and Engineers presented by President Obama (2010), the American Heart Association (AHA) Merit Award (2017), and the AHA Distinguished Scientist Award (2018). Professor Wu also received the inaugural AHA Joseph A. Vita Award (2015), which is presented to an investigator whose body of work published in the last 5 years has had transformative impact on basic, translational, or clinical cardiovascular research. Taken together, he is clearly a worthy recipient of the Bernard and Joan Marshall Distinguished Investigator Award. In his lecture, Professor Wu spoke about his pioneering work in 'Stem Cells and Genomics for Precision Medicine'. He began by discussing the advances in the field over the years, in particular the methodology for generating cardiomyocytes from human pluripotent stem cells, with the latest approaches now allowing $>90 \%$ cardiomyocyte purity following chemically defined differentiation from human pluripotent cells. He then discussed a number of cases and studies illustrating modelling of cardiac genetic disease in individual patients 
using iPSCs, with powerful data illustrating disease mechanisms and potential further therapeutic options. In an impressive and exciting grand tour of the possibilities of this approach, Professor $\mathrm{Wu}$ also discussed cardiac toxicity prediction, using iPSC-derived cardiomyocytes as a proxy to mimic the behaviour of adult heart in response to a range of drugs. This approach has been used by Professor Wu's group for prediction of both electrical repolarisation abnormalities as a cause for arrhythmias as well as cardiovascular toxicities associated with anticancer drugs, including anthracyclines, and newer agents, such as tyrosine kinase inhibitors. The breadth of examples and the depth of the science presented by Professor $\mathrm{Wu}$ were the highlight of the meeting and beautifully showcased the power of this approach for advancing precision medicine.

\section{References}

1. BSCR Autumn 2019 Meeting Abstracts. Cardiovasc Drugs Ther 2020. 33 (2) 261-274.

2. Lewis AJ, Tyler DJ, Rider O. Clinical cardiovascular applications of hyperpolarized magnetic resonance. Cardiovasc Drugs Ther. 2020. https://doi.org/10.1007/s10557-020-06942-w.

3. Mazzarotto F, Olivotto I, Walsh R. Advantages and perils of clinical whole-exome and whole-genome sequencing in cardiomyopathy. Cardiovasc Drugs Ther. 2020. https://doi.org/10.1007/s10557-02006948-4.

4. Preedy ME. Cardiac cyclic nucleotide phosphodiesterases: roles and therapeutic potential in heart failure. Cardiovasc Drugs Ther 2020.

5. Timm KN, Tyler DJ. The role of AMPK activation for cardioprotection in doxorubicin-induced cardiotoxicity. Cardiovasc Drugs Ther. 2020. https://doi.org/10.1007/s10557-020-06941-x.

Publisher's Note Springer Nature remains neutral with regard to jurisdictional claims in published maps and institutional affiliations. 